\title{
Cumulative identical spin rotation effects in collisionless trapped atomic gases
}

\author{
F. Piéchon, ${ }^{1}$ J.N. Fuchs, ${ }^{1}$ and F. Laloë ${ }^{2}$ \\ ${ }^{1}$ Laboratoire de Physique des Solides, CNRS UMR 8502, Univ. Paris-Sud, F-91405 Orsay, France and \\ ${ }^{2}$ Laboratoire Kastler Brossel, ENS, UPMC, CNRS; 24 rue Lhomond, F-75005 Paris, France
}

(Dated: November 7, 2018)

\begin{abstract}
We discuss the strong spin segregation in a dilute trapped Fermi gas recently observed by Du et al. with "anomalous" large time scale and amplitude. In a collisionless regime, the atoms oscillate rapidly in the trap and average the inhomogeneous external field in an energy dependent way, which controls their transverse spin precession frequency. During interactions between atoms with different spin directions, the identical spin rotation effect (ISRE) transfers atoms to the up or down spin state, depending on their motional energy. Since low energy atoms are closer to the center of the trap than high energy atoms, the final outcome is a strong correlation between spins and positions.
\end{abstract}

PACS numbers:

Spin waves in dilute gases were predicted at the beginning of the eighties [1, 2] and confirmed experimentally soon after [3, 4, 5]. They can be understood in two equivalent ways, either as a consequence of spin mean field [1], or in more microscopic terms as the cumulative result of the identical spin rotation effect (ISRE) an effect taking place during binary collisions between identical atoms [2]. Similarly, the Faraday effect is a rotation of the spin of photons that can be seen, either as a consequence of a macroscopic index of refraction, or as resulting from the accumulation of microscopic forward scattering events between photons and atoms.

Experiments with ultracold atomic gases have renewed the interest in spin waves. In 2002, a group at JILA [6] showed that, in an trapped ultracold atomic gas of bosons, the ISRE can result in a spontaneous spatial "segregation" of two atomic internal states $|1\rangle$ and $|2\rangle$ (equivalent to a pseudo-spin 1/2). Several groups then proposed a theoretical explanation of these observations, using either one-dimensional spin $1 / 2$ hydrodynamic [7] or kinetic 8, 9] equations. More recently, Du et al. from Duke University [10] did an experiment that explores the properties of spin waves in quantum gases of fermions $\left({ }^{6} \mathrm{Li}\right)$ in the collisionless Knudsen regime, while most previous experiments were performed in the hydrodynamic regime (see nevertheless [1]]). The spin segregation they observe is a hundred times larger and a hundred times slower than would be predicted by hydrodynamic theory. They call this spectacular effect "anomalous spin segregation" and suggest that its explanation may require "a modification of spin wave theory or possibly a new mechanism" for fermions.

The purpose of this letter is twofold. First we argue that no modification of spin wave theory is necessary to understand the experiment; the physical mechanism behind the observations is the usual ISRE. The difference between bosons and fermions is not essential; what is important is the collisionless regime. Second, we discuss why this collisionless regime, combined with the presence of a trap, gives access to unexpected and interesting new physics. In fact, one can observe the ISRE almost as in an ideal experiment, where a single spin polarized atom is sent through a target of a gas polarized in another direction, and where the spin direction of the outgoing atom is measured. Moreover, when the trap potential sends back the atom through the same target, the rotations of the spins are additive (the symmetry of ISRE is the same as that of the Faraday effect). As a result, one reaches situations where the spins of the atoms are correlated to their motional energies in the trap, instead of their positions; these situations are inaccessible in the hydrodynamic regime, where the value of the spin current is determined locally by a thermal average over many collisions.

We begin with a comparison between the JILA and Duke experiments. In the latter, the peak density $n(0)$ and the typical scattering length $a_{12}$ are respectively 15 and 20 times smaller than in the JILA experiment, making the diluteness factor $1 / n(0) a_{12}^{3}$ of the gas $10^{5}$ times larger. The differences can be expressed in terms of 5 relevant time scales: (1) the radial trap period $2 \pi / \omega_{\text {rad }}$; (2) the axial trap period $2 \pi / \omega ;(3)$ the average time between (lateral) collisions $\tau \sim\left(4 \pi a_{12}^{2} n(0) v_{T}\right)^{-1}$, where $v_{T} \equiv \sqrt{k_{B} T / m}$ is the thermal velocity, $T$ the temperature and $m$ the atomic mass; (4) the typical spin precession period in the external magnetic field $2 \pi /\left|\Omega_{0}\right| ;$ and (5) the typical precession period in the spin mean-field $\tau_{f w d} \sim m / \hbar\left|a_{12}\right| n(0)$. These time scales [in ascending order for the Duke exp.] are compared in the table:

\begin{tabular}{|c|c|c|c|c|c|}
\hline Time scales [in ms] & $2 \pi / \omega_{\text {rad }}$ & $2 \pi / \omega$ & $2 \pi / \mid \Omega_{0}$ & $\tau_{f w d}$ & $\tau$ \\
\hline Duke exp. [10] & 0.2 & 6.9 & 100 & 300 & 5000 \\
\hline JILA exp. [6] & 4 & 143 & 170 & 14 & 10 \\
\hline
\end{tabular}

where $a_{12} \sim-5 a_{0}$ and $\Omega_{0} / 2 \pi \approx-10 \mathrm{~Hz}$ are typical values for the Duke exp. [13]. The ISRE is then strong: $\tau / \tau_{f w d} \sim \hbar /\left(m v_{T}\left|a_{12}\right|\right) \gg 1$. In the JILA experiment, an hydrodynamic description was qualitatively correct because of the relatively small value of $\omega \tau \leq 1$. In the Duke experiment $\omega \tau \sim 4500$ : the gas is so dilute that its dynamics is well described in the collisionless limit (lateral collisions are ignored). On average, an atom oscillates 700 times in the axial trap between two collisions.

We base our analysis on the formalism used in [2] and [8], which gives a general frame for the study of non de- 
generate quantum gases. It distinguishes two effects of the interactions: mean field effects (forward scattering in collisions) and "real" collisions (lateral scattering), both with a full treatment of the effects of spin polarization and statistics. This leads to a kinetic equation with arbitrary position and momentum dependence. In the hydrodynamic limit, the system remains close to local equilibrium, momenta can be integrated out, and the spin current is simply proportional to the local gradient of magnetization. But we do not make such an assumption and keep a full dependence on the variables.

Consider a cigar-shaped harmonic trap with axial $(x$ direction) frequency $\omega / 2 \pi$ and radial ( $y$ and $z$ directions) frequencies $\omega_{\text {rad }} / 2 \pi$. As $\omega \ll \omega_{\text {rad }}$, an effective $1 \mathrm{D}$ description of the dynamics is possible. The peak density (per unit volume) $n(0)$ is related to the total number of atoms by $N=n(0)(2 \pi)^{3 / 2} x_{T} r_{\perp}^{2}$, where $x_{T} \equiv \sqrt{k_{B} T / m \omega^{2}}$ (resp. $\left.r_{\perp} \equiv \sqrt{k_{B} T / m \omega_{\text {rad }}^{2}}\right)$ is the characteristic atomic cloud size in the axial (resp. radial) direction. The two internal states $|1\rangle$ and $|2\rangle$ are treated as an effective spin $1 / 2$. The three relevant scattering lengths are $a_{12}, a_{11}$ and $a_{22}$; the corresponding coupling constants are $g_{i j} \equiv 4 \pi \hbar^{2} a_{i j} / m$. In spin space, $\mathbf{e}_{\|}$is the longitudinal unit vector, while $\mathbf{e}_{\perp 1}, \mathbf{e}_{\perp 2}$ correspond to the transverse directions. The spin couples to an external effective magnetic field $\Omega(x) \mathbf{e}_{\|}$, with an inhomogeneity along $x$ characterized by a curvature $\Omega^{\prime \prime}(0)$. Therefore, the magnetic field is taken to be $\Omega(x) \approx \Omega_{0} x^{2} / x_{T}^{2}$, where $\Omega_{0} \equiv \Omega^{\prime \prime}(0) x_{T}^{2} / 2$ is a characteristic spin precession frequency [14].

The kinetic equation for a spin $1 / 2$ non-degenerate atomic gas (fermions or bosons) is written in terms of a $2 \times 2$ operator $\hat{\rho}$ obtained by a Wigner transform of the density operator:

$$
\hat{\rho}(x, p, t)=\frac{1}{2}[f(x, p, t) \hat{I}+\mathbf{M}(x, p, t) \cdot \hat{\sigma}]
$$

Equivalently, we can reason in terms of the phase space density $f$ and spin density M. In the Duke experiment, the gas is non-degenerate $\left(T \approx 27 \mu \mathrm{K} \gg T_{F} \approx 7 \mu \mathrm{K}\right)$. As in [8] we make some approximations: both the density mean-field $\left|g_{12}\right| n(0) / 4 \approx h \times 2 \mathrm{~Hz}$ and the Stern and Gerlach segregation energies $\hbar\left|\Omega_{0}\right| \approx h \times 10 \mathrm{~Hz}$ are negligible when compared to the harmonic confining potential $m \omega^{2} x_{T}^{2} / 2=k_{B} T / 2 \approx h \times 280 \mathrm{kHz}$. Since lateral collisions are a small perturbation, we treat them in the simplest relaxation time approximation. Here a single coupling constant $g_{12} \neq 0$ appears, instead of three, because $g_{11}=g_{22}=0$ results from the Pauli exclusion principle (collisions only occur between atoms in different spin states). We then obtain the equations [8]:

$$
\begin{aligned}
d_{t} f \equiv\left(\partial_{t}+p \partial_{x}-x \partial_{p}\right) f & \simeq-\frac{f-f^{e q}}{\tau} \\
d_{t} \mathbf{M}-\left[\Omega(x) \mathbf{e}_{\|}+\epsilon g \mathbf{m}\right] \times \mathbf{M} & \simeq-\frac{\mathbf{M}-\mathbf{M}^{e q}}{\tau}
\end{aligned}
$$

where $\epsilon=-1$ for fermions (and +1 for bosons); the superscript $^{e q}$ denotes local equilibrium phase space densities; the dimensionless coupling constant $g$ is defined as $g \equiv g_{12} n(0) / 2 \hbar \omega$. Dimensionless units have been used: lengths are measured in units of $x_{T}$, momenta in units of $p_{T} \equiv m v_{T}$, angular frequencies in units of $\omega$, times in units of $1 / \omega$, and phase space densities $f$ and $\mathbf{M}$ in units of $n(0) / p_{T} \sqrt{2 \pi}$. The density $n$ and the spin density $\mathbf{m}$ [both in units of $n(0)$ ] are defined as:

$$
n(x, t)=\int \frac{d p}{\sqrt{2 \pi}} f \text { and } \mathbf{m}(x, t)=\int \frac{d p}{\sqrt{2 \pi}} \mathbf{M}
$$

such that $1=\int d x n / \sqrt{2 \pi}$ [and e.g. $\mathbf{e}_{\perp 1}=\int d x \mathbf{m} / \sqrt{2 \pi}$ if the gas is fully polarized in the $\mathbf{e}_{\perp 1}$ direction]. The total density $n=n_{1}+n_{2}$ and the longitudinal spin density $m_{\|}=n_{2}-n_{1}$ are related to the internal state populations, while the transverse spin density $\mathbf{m}_{\perp}$ to their coherences.

These equations are now solved numerically. Since the experiment starts with a $\pi / 2$ pulse which suddenly transfers the spins to the transverse plane, the initial distributions are $f_{0}(x, p)=\exp \left[-\left(x^{2}+p^{2}\right) / 2\right]$ and $\mathbf{M}_{0}(x, p)=$ $f_{0}(x, p) \mathbf{e}_{\perp 1}$. As $f_{0}$ solves the kinetic equation, the total density does not evolve $n(x)=\exp \left(-x^{2} / 2\right)$ and the dynamics after the pulse can be expressed in terms of $\mathbf{M}$ only. The 3 dimensionless parameters appearing in the kinetic equations are taken as $\epsilon g=0.021$ [corresponding to $a_{12}=-4.6 a_{0}$ and $\left.\epsilon=-1\right], \Omega_{0}=-0.07$ and $1 / \tau=2 \times 10^{-4}$. Fig. 1 compares the results with those plotted in fig. 2c of [10]: both the maximum segregation time $\left(t_{\max } \sim 300 \mathrm{~ms}\right)$ and amplitude $\left(m_{\|}\left(0, t_{\max }\right) / n(0) \sim 30 \%\right)$ are in good agreement with the experimental data. Note also that the sign of the segregation is predicted correctly. It is clear that standard theory with a transport equation treated in the collisionless regime explains the observations without introducing any new mechanism. The system eventually relaxes to equilibrium on the time scale of a few $\tau$, which can be very slow indeed because $\tau \sim 5 \mathrm{~s}$. Calculations for other values of $a_{12}$ are in fair agreement with the experimental data. In particular, changing the sign of $a_{12}$, of $\Omega_{0}$ or of $\epsilon$ simply reverses the role of the two internal states. When $a_{12} \approx 0$, no significant segregation takes place. Fig. 2 shows the longitudinal spin density profile at saturation and a good agreement with the experiment results [10].

In order to better understand the numerical results of the previous section, approximate analytical solutions of the linearized $[|g|<<1]$ collisionless kinetic equations are now provided. In the absence of lateral collisions, the (axial) motional energy of an atom $E=\left(x^{2}+p^{2}\right) / 2$ [in units of $k_{B} T$ ] is a constant of the motion and $\mathbf{M}(x, p, t)$ conserves its norm. We therefore write $M_{\|}=f_{0} \sin \theta$, $M_{\perp}=M_{\perp 1}+i M_{\perp 2}=f_{0} \cos \theta e^{i \phi}$, which define the transverse $\phi(x, p, t)$ and longitudinal $\theta(x, p, t)$ precession angles in phase space. Accordingly the initial conditions are $\theta(x, p, 0)=0$ and $\phi(x, p, 0)=0$. Eq. (3) translates into coupled equations of motion for $\theta$ and $\phi$ :

$$
\begin{aligned}
& d_{t} \phi=\Omega+\epsilon g\left(\langle\sin \theta\rangle-\frac{\sin \theta}{\cos \theta} \operatorname{Re}\left\{e^{i \phi}\left\langle\cos \theta e^{-i \phi}\right\rangle\right\}\right)(5) \\
& d_{t} \theta=\epsilon g \operatorname{Im}\left\{e^{i \phi}\left\langle\cos \theta e^{-i \phi}\right\rangle\right\}
\end{aligned}
$$




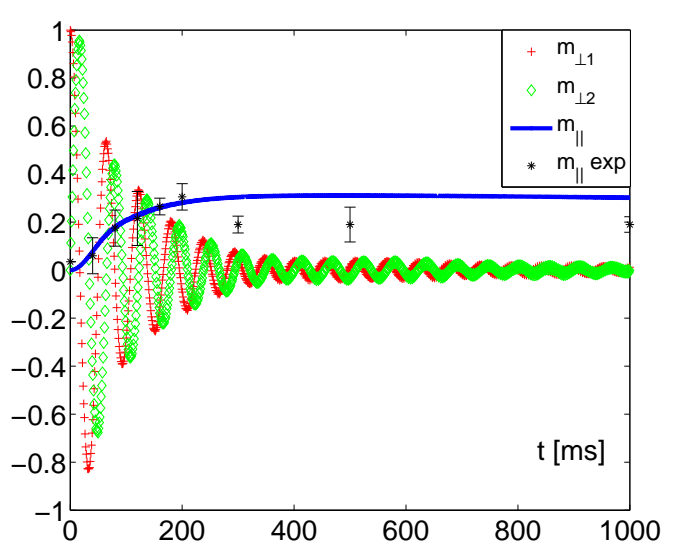

FIG. 1: Spin density at the center of the trap as a function of time $\mathbf{m}(x=0, t)$ [in units of $n(0)$ ] for $\epsilon g=0.021, \Omega_{0}=-0.07$ and $1 / \tau=2 \times 10^{-4}$. We obtain a good agreement with the measurements of $m_{\|}=n_{2}-n_{1}$, taken from fig. 2c of [10] and shown here with their error bars: both the amplitude and the time constants of the spin segregation are well reproduced.

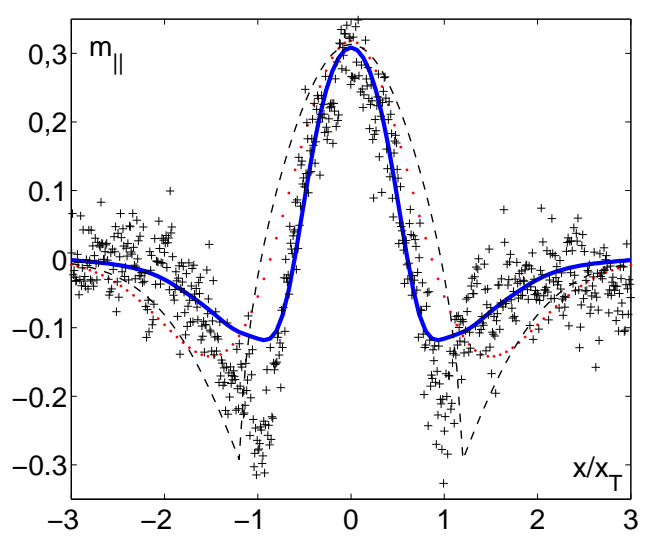

FIG. 2: Numerical results (full line) for the longitudinal spin density at maximum segregation time $m_{\|}\left(x, t_{\max }\right)$ [in units of $n(0)]$ as a function of position; the parameters are the same as in fig. 1 and $x_{T}=210 \mu \mathrm{m}$. These results are in very good agreement with the measurements of [10] (crosses) at the trap center and edges, without any adjustable parameter. The discrepancy close to $x / x_{T} \approx \pm 1$ may be attributed to a small population imbalance in the experiment $(\sim 4 \%$ as estimated from the area under the experimental profile). The results of two approximations are also shown (dotted and dashed lines); see text for more details.

where the momentum average is defined as $\langle A\rangle \equiv$ $\int \frac{d p}{\sqrt{2 \pi}} f_{0} A$ for any function $A(x, p, t)$. To first order in $g$, we can ignore the second term in eq. (5) and assume $\cos \theta \simeq 1$ in eq. (6) (furthermore $M_{\|} \simeq f_{0} \theta$ and $\left.M_{\perp} \simeq f_{0} e^{i \phi}\right)$. These linearized equations can be solved exactly [12]. Here we just quote the results for the trans- verse spin precession angle $\phi(x, p, t)$ :

$$
\phi=\Omega_{0}\left[\frac{1}{2} \gamma_{+}(t) x^{2}+\frac{1}{2} \gamma_{-}(t) p^{2}-\gamma_{0}(t) x p\right]
$$

where $\gamma_{ \pm}(t) \equiv t \pm \frac{\sin 2 t}{2}, \gamma_{0}(t) \equiv \sin ^{2} t$. The longitudinal spin density $m_{\|}(x, t)$ is:

$$
m_{\|}=\epsilon g \int_{0}^{t} d t^{\prime} \frac{e^{-\frac{Z}{\Lambda X} x^{2}}}{\sqrt{X} \Lambda^{1 / 4}} \sin \left[\frac{1}{2} \arctan \frac{Y}{X}-\frac{Z}{\Lambda X} \frac{Y}{X} x^{2}\right]
$$

where $Z\left(t^{\prime}\right) \equiv 1+\Omega_{0}^{2}\left(\gamma_{0}^{2}\left(t^{\prime}\right)+\gamma_{-}^{2}\left(t^{\prime}\right)\right), \quad Y\left(t, t^{\prime}\right) \equiv$ $2 s \Omega_{0}\left(\gamma_{0}\left(t^{\prime}\right) c-\gamma_{-}\left(t^{\prime}\right) s\right), X\left(t, t^{\prime}\right) \equiv 1+s^{2}+\Omega_{0}^{2}\left(\gamma_{0}\left(t^{\prime}\right) s+\right.$ $\left.\gamma_{-}\left(t^{\prime}\right) c\right)^{2}, \Lambda\left(t, t^{\prime}\right) \equiv 1+\left(\frac{Y}{X}\right)^{2}$, using the shorthand notation $s \equiv \sin \left(t-t^{\prime}\right)$ and $c \equiv \cos \left(t-t^{\prime}\right)$.

Three regimes can be distinguished: (i) for times smaller than the trap period $(t \ll 1)$, at a fixed position $x$, the transverse phase difference between two atoms with momentum $p$ and $p^{\prime}$ is dominated by the $x-p$ correlation term $\gamma_{0}(t)$ in eq. (77): $\phi(x, p, t)-\phi\left(x, p^{\prime}, t\right) \simeq$ $\Omega_{0} t^{2} x\left(p-p^{\prime}\right) \ll 1$. This is the regime leading to correlation between velocity and transverse spin and giving:

$$
m_{\|}(x, t) \approx \operatorname{\epsilon gn}(x)^{2}\left(1-2 x^{2}\right) 2 \Omega_{0} \frac{t^{4}}{4 !}
$$

as already found in [8, 9]. For the Duke exp., this behavior is not dominant since it occurs only at very short times [smaller than $\sim 1 \mathrm{~ms}$ ]. However, for times much longer than the trap period $t \gg 1$ there is a transition to an energy dominated regime where $\phi(x, p, t) \simeq$ $\phi(E, t) \simeq \Omega_{0} E t$, where $\Omega_{0} E$ emerges as an effective transverse precession frequency. (ii) A second regime occurs for $1 \ll t \ll 1 /\left|\Omega_{0}\right|$ where the phase difference $\phi(E, t)-\phi\left(E^{\prime}, t\right) \simeq \Omega_{0} t\left(E-E^{\prime}\right) \ll 1$ is small - this is the regime of correlation between motional energy and transverse spin. Averaging over fast axial oscillations of small amplitude, we obtain the approximate result:

$$
m_{\|}(x, t) \approx-\epsilon g n(x)^{4 / 3}\left(1-\frac{4}{3} x^{2}\right) \frac{\sqrt{2}}{3 \sqrt{3}} \Omega_{0} t^{2}
$$

This gives the dominant contribution to the spin segregation in the Duke experiment. Note that there is a sign inversion as compared to regime (i) and that the scaling is now $t^{2}$ instead of $t^{4}$ [15]. The typical time for triggering the spin segregation is $t_{\text {trig }} \sim 1 / \sqrt{\left|g \Omega_{0}\right|}$. The dotted line of Fig. 2 shows a plot of (10) for $t \sim 1 /\left|\Omega_{0}\right|$. (iii) The third regime is an ergodic regime: when $t \gg 1 /\left|\Omega_{0}\right|$, the phase difference between high and low energy atoms becomes large $\phi(E, t)-\phi\left(E^{\prime}, t\right) \simeq \Omega_{0} t\left(E-E^{\prime}\right) \gg 1$, therefore saturation occurs. However, the saturation is not captured by the linearized equations as it is actually related to norm conservation and to the non-linearity of the kinetic equations [12]. By comparing the numerics to the analytical solution of the linearized equations, we find that they agree for the first $\sim 100 \mathrm{~ms}$.

A simple model captures the essence of the phenomenon. Assume that we can divide the atoms into two 
classes, the "hot atoms" with some motional energy (for instance $3 k_{B} T$ ) and the "cold atoms" with some lower energy (for instance $k_{B} T / 2$ ). All the atoms oscillate quickly in the trap so that the precession rate of their spins depends on the average of the longitudinal magnetic field along their trajectory [16]. Since the hot atoms go further away from the center of the trap, their transverse spin component rotates at a different rate than for the cold atoms. Now, when a hot atom crosses the cloud of cold atoms, which has a different spin direction, its spin experiences a rotation due to the ISRE in forward scattering. This gives to the hot atom a longitudinal spin component that depends on the sign of the ISRE, while the cold atoms acquire the opposite component. The effect is cumulative: each time the hot atom crosses the cloud forwards and backwards, the rotations of its spin are additive. After some time, a large fraction of the hot atoms is transferred towards one spin state, a large fraction of the cold atoms to the other. A situation where the two classes of atoms have antiparallel longitudinal spins is stable as long as lateral collisions are ignored, since ISRE does not affect antiparallel spin directions, and since any phase space distribution that depends only on the energy is time invariant. Finally, because the atoms explore a region of space that depends on their energy, the spin state of the cold atoms is dominant at the center of the trap, the spin state of the hot atoms at the edges.

To illustrate how this spatial separation arises from spin-energy correlations, we arbitrarily separate the atoms in two equal groups, with axial motional energy larger or smaller than $k_{B} T \ln 2$. The difference in density profile between cold $n_{<}(x)$ and hot $n_{>}(x)$ atoms is then easily obtained in terms of the error function:

$$
n_{<}-n_{>}=n(x)\left[2 \operatorname{Re}\left\{\operatorname{Erf}\left[\sqrt{\ln 2-x^{2} / 2}\right]\right\}-1\right]
$$

If the correlation $c$ between longitudinal spin and motional energy is not $100 \%$, but say $c=60 \%$, this result is reduced by the corresponding factor. The dashed line in fig. 2 is a plot of $0.6\left(n_{<}-n_{>}\right)$according to (11); a comparison with the other curves shows that this model already gives a reasonable understanding of the profile.

In conclusion, two major features of the new phenomenon are: a cumulative effect of the ISRE producing a strong correlation between motional energies and longitudinal spin directions, and its stability over long times. The nature of the effect is more ballistic than hydrodynamic. The shape of the profile depends on the properties of the trap, not of the interactions: any phase space distribution of the internal states that depends only on the energy remains invariant under time evolution in the trap. ISRE therefore plays a crucial role in creating the profile, but not in maintaining it.

Acknowledgements. - We thank J.E. Thomas and X. $\mathrm{Du}$ for sharing their data with us and A. Amaricci for help with the numerics.
[1] E.P. Bashkin, JETP Lett. 33, 8 (1981); JETP 60, 1122 (1984).

[2] C. Lhuillier and F. Laloë, J. Physique 43, 197 (1982); ibid 43, 225 (1982).

[3] B.R. Johnson, J.S. Denker, N. Bigelow, L.P. Levy, J.H. Freed and D.M. Lee, Phys. Rev. Lett. 52, 1508 (1984).

[4] P.J. Nacher, G. Tastevin, M. Leduc, S.B. Crampton and F. Laloë, J. Phys. Lett. 45, L-441 (1984); 46, 249 (1985).

[5] W.J. Gully and W.J. Mullin, Phys. Rev. Lett 52, 1810 (1984).

[6] H.J. Lewandowski, D.M. Harber, D.L. Whitaker and E.A. Cornell, Phys. Rev. Lett. 88, 070403 (2002).

[7] M. Ö. Oktel and L.S. Levitov, Phys. Rev. Lett. 88, 230403 (2002).

[8] J.N. Fuchs, D.M. Gangardt, and F. Laloë, Phys. Rev. Lett. 88, 230404 (2002); Eur. Phys. J. D 25, 57 (2003).
[9] J.E. Williams, T. Nikuni, and C.W. Clark, Phys. Rev. Lett. 88, 230405 (2002).

[10] X. Du, L. Luo, B. Clancy, and J.E. Thomas, Phys. Rev. Lett. 101, 150401 (2008).

[11] N.P. Bigelow, J.H Freed and D.M. Lee, Phys. Rev. Lett. 63, 1609 (1989).

[12] F. Piéchon and J.N. Fuchs, in preparation.

[13] In the Duke experiment, the scattering length $a_{12}$ could be tuned, but the precession frequency $\Omega_{0}$ was fixed; in the JILA experiment, the reverse was true.

[14] The relation between $\Omega_{0}$ and $\delta \Omega$ defined in [8] is $\Omega^{\prime \prime}(0)=$ $2 \Omega_{0} / x_{T}^{2}=\delta \Omega / x_{T}^{2}$. By definition $\hbar \Omega \equiv V_{2}-V_{1}$, see [8].

[15] The sign of the segregation in [10] (resp. [6]) corresponds to that of the $t^{2}$ (resp. $t^{4}$ ) law.

[16] In a quadratic trap, the rate is proportional to the energy since $\int d t \Omega[x(t)] / 2 \pi=\Omega_{0} E$. 